\title{
A new psychophysical ratio scaling technique: Random production*
}

\author{
WILLIAM P. BANKS $\dagger$ \\ Pomona College, Claremont, Calif. 91711
}

A new technique for psychophysical scaling, capable of generating a ratio scale for any perceptual continuum that can be used as a response continuum, is described and used to scale subjective force on a handgrip, subjective areal extent, and subjective intensity of electric shock. For these continua, the technique yields psychophysical scales that are very well fit by power functions, but the exponents are somewhat smaller than the exponents obtained for these continua in magnitude estimation scaling.

This paper is a preliminary report of a new technique for psychophysical scaling and of scales for three continua obtained with it. The technique can be applied to any psychophysical continuum whose quantities can be generated by a $\mathrm{S}$. If a single, seemingly plausible, assumption about Ss' behavior in the task is true, then the technique gives a ratio scale of the subjective continuum.

The technique poses a fairly simple task for the $\mathrm{S}: \mathrm{He}$ is asked to generate random quantities on a psychophysical continuum in such a way that they are spaced evenly (uniformly) over it. For illustration of the task, consider how the continuum of subjective force as exerted on a hand dynamometer would be scaled. The $S$ is first given a hand dynamometer and allowed to give it a number of weak and strong squeezes so that he has some "feel" for the continuum. Then he is asked to think of the continuum as a graded continuous series of squeezes, going from the very weakest to the strongest he can generate. He is then told to give a number of separate squeezes of various strengths on the handgrip in such a way that the squeezes he gives sample the continuum evenly. That is, he is told to be sure to give as many strong as weak squeezes and to try to allow every region on his subjective continuum of squeeze strength to have an equal chance of being produced. After each squeeze the $S$ relaxes his hand, the $E$ reads and resets the dynamometer scale, and the $S$ produces another random squeeze.

An informal experiment revealed the somewhat trivial

\footnotetext{
*This research was funded by a Pomona College research grant, and some of the work was done while the author was supported by a Pomona College summer fellowship. The handgrip scale was obtained at the John B. Pierce Foundation Laboratory in New Haven, Connecticut. The other research, as well as the writing, was done while the author was visiting the Psychology Department at Stanford University. The generosity Psychology Department at Stanford University. The generosity
of both institutions in providing facilities and apparatus is gratefully appreciated.

+Requests for reprints should be sent to William P. Banks, Department of Psychology, Pomona College, Claremont, California 91711.
}

fact that Ss could sample squeeze intensities fairly uniformly up to their limit of strength when they could see the scale on the dynamometer. But what would happen when they could not see the scale? In this case they must monitor their samples on their internal representation of the continuum, and the samples would therefore be uniform over the subjective rather than the objective scale. From a purely intuitive point of view, it seems reasonable that productions that are uniformly sampled from the subjective continuum would give a picture of how the objective continuum maps on to the subjective one. This is so because physically equal intervals along the objective continuum should be sampled in proportion to their psychological size: If the interval between 5 and $10 \mathrm{~kg}$ seems subjectively as wide as the interval between 10 and $15 \mathrm{~kg}$, then the two intervals should, on the average, contain the same number of random productions.

It is possible to go far beyond intuitive speculations and construct a ratio scale of the subjective continuum from the random productions. The technique for generating a ratio scale of the subjective continuum uses the order statistic means (Hogg, 1969) of the random productions. To take the mean order statistics, the physical value of each S's set of $n$ random productions is put in order from smallest to largest, then the mean for each ordinal position is taken across Ss. Assuming that the subjective continuum is finite and that sampling on the subjective continuum is uniform, then the probability density function of $Y_{k}$, the $k$ th of the $n$ order statistics is:

$$
g_{k}(y)=\frac{n !}{(k-1) !(n-k) !} \cdot(y)^{k-1}(1-y)^{n-k}
$$

The expected value of $Y_{k}$ is

$$
E\left[Y_{k}\right]=\int_{0}^{1} y_{k}(y) d y
$$

where the subjective scale extends from 0 to 1 .

It follows that

$$
\begin{aligned}
E\left[Y_{k}\right] & =\int_{0}^{1} \frac{n !}{(k-1) !(n-k) !} y^{k}(1-y)^{n-k d y} \\
& =\frac{n !}{(k-1) !(n-k) !} \cdot \frac{\Gamma(k+1) \Gamma(n-k+1)}{\Gamma(n+2)} \\
& =\frac{n !}{(k-1) !(n-k) !} \cdot \frac{k !(n-k) !}{(n+1) !} \\
& =k \frac{1}{n+1}
\end{aligned}
$$




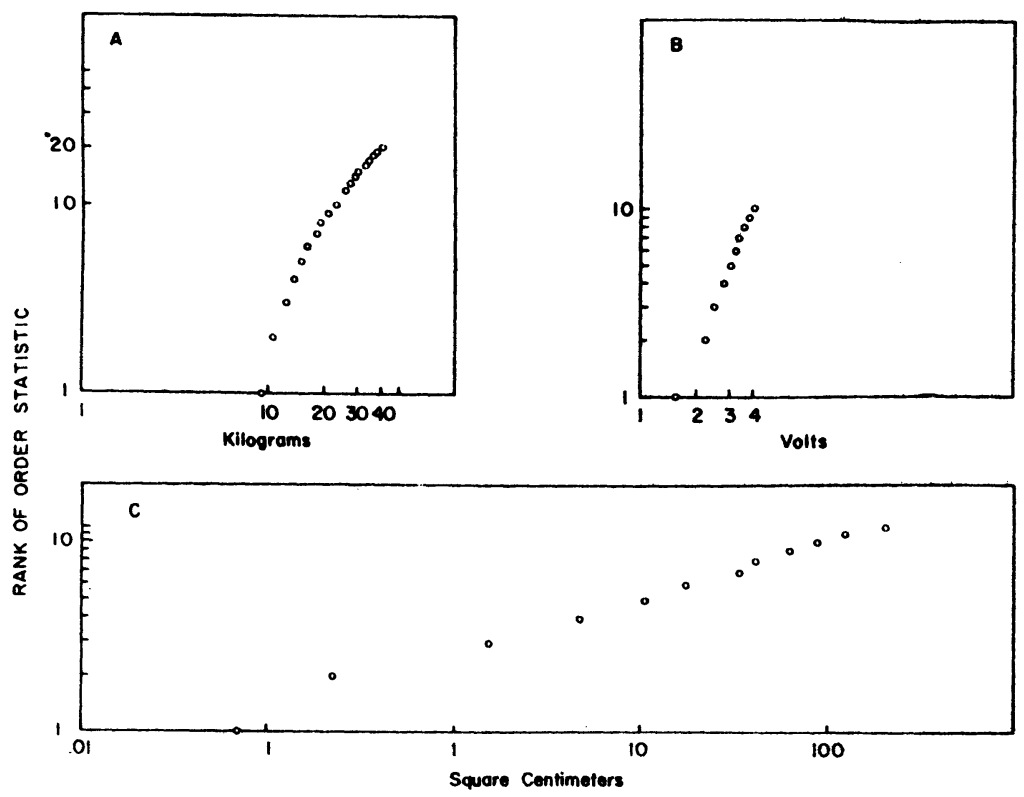

Fig. 1. Three random production scales: (a) rank vs magnitude (kilograms) of order statistics for handgrip force; (b) rank vs magnitude (volts) of order statistics for intensity of electric shock; (c) rank vs magnitude (centimeters squared) of order statistics for subjective size of squares. All in $\log -\log$ coordinates.

MAGNITUDE OF ORDER STATISTIC

This result says that the mean of the kth order statistic will be $k$ units of size $1 /(n+1)$ from the bottom of the subjective scale. Consequently, the $n$ order statistic means will be evenly spaced on the subjective continuum with a unit difference proportional to $1 /(n+1)$. Since the subjective continuum was arbitrarily assumed to go from 0 to 1 , the size of this unit is not known. If the continuum went from 0 to $\mathrm{N}$, the unit would be $N /(n+1)$. Thus, if the 0 point of the subjective scale is known, the spacing of the order statistics specifies a ratio scale of the continuum with an arbitrary unit.

\section{SCALES FOR AREA, HANDGRIP SQUEEZE, AND SHOCK}

Three psychophysical continua were scaled with the random production technique: subjective area of squares, subjective strength of hand dynamometer squeeze, and subjective intensity of a $60-\mathrm{Hz}$ electric shock.

\section{Handgrip}

The random squeezes were obtained using the procedure discussed above, with 12 males aged 19-24 years as Ss, each contributing 20 random squeezes on a precision hand dynamometer that had less than $1 / 2 \mathrm{~cm}$ of excursion per $100 \mathrm{~kg}$ of force. Each S's 20 squeezes were recorded in kilograms and put in order from smallest to largest, and geometric means of the squeeze values at each ordinal position were used to estimate the magnitude of the 20 order statistics.

In Fig. 1a the rank of the order statistics is plotted against their magnitude in kilograms. Since the 20 order statistics are spaced evenly on the subjective continuum, the function traced out in Fig. 1a shows how handgrip squeezes measured in kilograms must be adjusted so that they are spaced evenly in subjective units. That is to say, the function shown is the psychophysical mapping function for force exerted on a handgrip. Plotted in log-log coordinates, this function has the appearance of a magnitude estimation power function in need of a threshold correction (Marks \& Stevens, 1968). If a threshold of $7 \mathrm{~kg}$ is assumed, the function becomes linear in $\log -\log$ coordinates, with a slope (i.e., power function exponent) of $1.09, \mathrm{r}^{2}=.994$

The exponent found here for handgrip is much smaller than the 1.7 usually found with magnitude estimation (J. C. Stevens \& Mack, 1959), and it was decided to try the technique on several other continua with very different exponents to see if their magnitude estimation exponents were similarly underestimated by random production scales. Subjective area and intensity of electric shock were chosen as continua greatly different in exponent and easy to scale with random production.

\section{Shock}

For random productions of electric shock, Ss controlled a variable transformer whose $60-\mathrm{Hz}$ output went through a step-down transformer and then to two drinking glasses containing a $5 \%$ solution of $\mathrm{NaCl}$ in water. The Ss operated the variable transformer with one hand and had one finger of the other hand in each of the two glasses of electrolyte to receive the shock. Instructions and procedure were much the same as for the handgrip experiment; Ss were 11 males (undergraduates, graduate students, and faculty members at Stanford University); and each $S$ produced 10 random subjective intensities of electric shock. In most cases Ss removed their fingers from the electrolytic baths while the $\mathrm{E}$ read the voltage, and they reset the transformar to zero before reinserting their fingers to monitor the next random production.

Figure 1b shows how the subjective intensity of shock (rank of order statistic) rises with the geometric mean of produced RMS voltage. This scale is extremely well fit with a power function whose exponent is $2.49\left(\mathrm{r}^{2}=.989\right)$. In addition, the power function fit to each of the $11 \mathrm{Ss}^{\prime}$ unaveraged sets of 10 productions was excellent, and every $S$ produced an accelerated scale. Since $E=I \cdot R$, the present physical scale (volts) differs from the physical scale of magnitude estimation studies (amperes) by an arbitrary constant factor (the Ss' geometric mean skin resistance). Both volts and amperes should, therefore, lead to the same psychophysical exponent. Thus, to judge by magnitude estimation scales (Stevens, Carton, \& Shickman, 1958), the present exponent should have been closer to 3.5 than to 2.5 .

\section{Area}

Subjective area was scaled by having Ss draw squares on sheets 
of $8 \frac{1}{2} \times 11$ in. unlined paper. Instructions similar to those for random productions of shock and handgrip squeeze were given. The Ss were asked to draw squares which randomly sampled the range of apparent sizes that could be drawn on a sheet of the paper. One sheet was provided for each random square, and the sheet was removed from S's view after he had drawn a square on it. The Ss were 12 male and female Stanford University undergraduates, who performed in this experiment while taking a rest break in an unrelated experiment. Each $\mathrm{S}$ drew 12 random squares, and the area of each of these was computed as the product of its average length and average width measurement in centimeters (it was necessary to take average measurements because the squares were drawn freehand).

Figure 1c gives the random production scale for subjective area, where the magnitude of the order statistics was estimated by the geometric mean. This function is well fit by a power function with an exponent of $.30\left(r^{2}=.992\right)$. It is an extremely decelerated function, but the fit of a logarithmic function gives an $\mathrm{r}^{2}$ of only .90 , over which the power function fit is clearly superior. Again, the random production exponent is considerably smaller than the magnitude estimation exponent for the continuum, which is around .7 or .8 (Stevens \& Guirao, 1963; Teghtsoonian, 1965).

\section{DISCUSSION}

The fact that random production seems consistently to give psychophysical scales with exponents below those found with magnitude estimation has several interpretations. First, it might be argued that, because of the difficulty or pain involved, Ss avoid the larger or more intense stimuli on the continuum and thereby produce an artificially overcompressed scale. This argument is, however, at odds with the present results. Certainly, if Ss avoided the larger productions to avoid the pain or effort they entail, the shock scale should be the most compressed, the handgrip scale the next most compressed, and the area scale the least compressed. The results are exactly the opposite of this ordering, as is seen in Fig. 1a-c. A more sophisticated version of this argument would be that the random production scale of shock should underestimate its magnitude estimation exponent most, the handgrip scale the next most, and the area scale the least. This, too, is contrary to the results: The random production exponent for shock is $71 \%$ of the size of the magnitude estimation exponent for shock, the random production exponent for handgrip force is $65 \%$ of the magnitude estimation exponent, and the random production exponent for area is only about $40 \%$ of the magnitude estimation exponent.

S. S. Stevens (1971) might classify the random production technique as a "partitioning" scaling method. Since the partitioning methods typically yield a "virtual" exponent that is smaller than the "actual" exponent obtained through magnitude estimation, the undersized exponents obtained by random production are to be expected by this classification. However, the agreement with Stevens's classification does not in itself constitute an explanation of the relatively small exponents found in random production. At best, this agreement tells us to look for the same explanation for the size of exponent obtained in random production as in the partitioning techniques, such as equisection or interval estimation.

Finally, the random production scales presented here depend on Ss following the instructions to sample uniformly on the subjective continuum, and other assumptions about the sampling distribution would generate different psychophysical scales from the present results. However, the uniform distribution seems so easy for Ss to understand and so intuitively plausible that it is probably an excellent first approximation to the distribution they are following. In any event, it is unlikely that deviation from uniformity of sampling can account for the overcompression of the scales relative to magnitude estimation scales. The most plausible nonuniform sampling hypothesis, that Ss systematically avoid the most painful stimuli, is contradicted by the data, and other plausible nonuniform hypotheses, if they exist, would have to be tested as they are discovered. Also, it seems unlikely that the power function form of the subjective scale found by random production is the result of a nonuniform sampling operation on a subjective continuum spaced according to some function other than a power function. Of course, it is possible that Ss sample nonuniformly on a nonpower function scale, but the present assumption of uniform sampling on a power function scale is much simpler. It would be surprising if the Ss hit on just the right nonuniform sampling distribution to convert their nonpower function scales into power functions.

\section{REFERENCES}

Hogg, R. V. Introduction to mathematical statistics. New York: MacMillan, 1969

Marks, L. E., \& Stevens, J. C. The form of the psychophysical function near threshold. Perception \& Psychophysics, 1968, 4, 315-318.

Stevens, J. C., \& Mack, J. D. Scales of apparent force. Journal of Experimental Psychology, 1959, 58, 405-413.

Stevens, J. C., Mack, J. D., \& Stevens, S. S. Growth of sensation on seven continua as measured by force of handgrip. Journal of Experimental Psychology, 1960, 59, 60-67.

Stevens, S. S. Issues in psychophysical measurement. Psychological Review, 1971, 78, 426-450.

Stevens, S. S., Carton, A. S., \& Shickman, G. M. A scale of apparent intensity of electric shock. Journal of Experimental apparent intensity of electric sho

Stevens, S. S., \& Guirao, M. Subjective scaling of length and area and the matching of length to loudness and brightness. Journal of Experimental Psychology, 1963, 66, 177-186.

Teghtsoonian, M. The judgment of size. American Journal of Psychology, 1965, 78, 392-402.

(Received for publication January 19, 1973.) 